\title{
A randomized, crossover trial comparing the efficacy and safety of fesoterodine and extended- release oxybutynin in children with overactive bladder with 12-month extension on fesoterodine: The FOXY study
}

Sophie Ramsay, MD*; Élizabeth Naud, MD*; David Simonyan, MSc; Katherine Moore, MD; Stéphane Bolduc, MD

Division of Urology, CHU de Québec - Université Laval Research Center, Quebec City, QC, Canada

${ }^{*}$ Equal contributors

Funding: Supported by an IIR grant by Pfizer Canada: WI191464

Acknowledgements: The authors would like to thank the FOXY study investigators, and all patients and their parents/legal representatives who took part in the study. Special thanks to Louise Gosselin, study coordinator, and Marie-France Nolin, research nurse for their continuous effort and support to patients and their family. Further thanks to our Drug Safety Monitoring Board (DSMB) and colleagues Dr. Marie-Claude-Roy, pediatric nephrologist, and Dr. Philippe Chetaille, pediatric cardiologist, for their support.

Cite as: Can Urol Assoc J 2020 January 20; Epub ahead of print. http://dx.doi.org/10.5489/cuaj.6247

Published online January 20, 2020

$* * *$

\section{Abstract}

Introduction: We sought to assess and compare safety and efficacy of fesoterodine and oxybutynin extended-release in the treatment of pediatric overactive bladder (OAB).

Methods: We conducted a non-inferiority, randomized, double-blind, crossover trial comparing fesoterodine 4-8 $\mathrm{mg}$ and oxybutynin 10-20 mg QD in children with OAB aged 5-14 years (20152018). Every child received the first medication for eight weeks, followed by crossover to the second antimuscarinic after a three-days washout. Dose up-titration was possible at mid-course. Patients could enter a fesoterodine 12-month extension. Endpoints were assessed through changes on voiding diaries, Patient's Perception of Bladder Condition score (PPBC), adverse events, vital signs, electrocardiogram, post-void residual, urinalysis, and blood tests. The Wilcoxon rank sum and Wilcoxon signed rank tests were used for statistical analysis.

Results: A total of 62 patients were randomized (two early dropouts). Expected class effects (dry mouth/constipation) were present but no significant difference was observed. There was a 10.1 beats/minute increase in heart rate with fesoterodine $(\mathrm{p}<0.01)$ (oxybutynin -1.9 beats $/ \mathrm{min} ; \mathrm{p}=\mathrm{ns})$. No life-threatening or serious adverse events occurred. Efficacy was similar for both drugs. Bladder capacity improved over the 16 months of the study; baseline capacity of $125 \mathrm{~mL}(44.5 \%$ expected 
bladder capacity for age [\%EBC]) to $171 \mathrm{~mL}(53.9 \% \mathrm{EBC})$ at the end of the extension phase. No clinical or statistical difference was shown between efficacy measures for fesoterodine or oxybutynin. Conclusions: The use of fesoterodine or oxybutynin appears as safe and effective for the treatment of $\mathrm{OAB}$ in children. Based on our study, long-term treatment to achieve the ultimate goal of urinary continence is needed in this population.

\section{Introduction}

Overactive bladder $(\mathrm{OAB})$ is defined as: "urinary urgency, usually with frequency and nocturia, with or without urge incontinence, in the absence of UTI or other pathology." [1] Lower urinary tract symptoms (LUTS) are a burdensome healthcare issue, accounting for up to $40 \%$ of pediatric-urology visits, [2] and OAB has an estimated prevalence of $15-20 \%$ in children. [3-4] LUTS are recognized as having harmful impact on children's quality of life, including poor self-esteem, social isolation and behavioural changes. [5]

Conservative measures are the backbone of $\mathrm{OAB}$ treatment and should not be neglected. When they are insufficient to treat symptoms, other options should be considered. Antimuscarinics are the firstline pharmacologic OAB therapy, [6] but their use has not been as extensively studied in children. Oxybutynin (Oxy) immediate-release (IR) has long been the sole FDA-approved antimuscarinic for pediatric OAB treatment. Its treatment-emergent adverse reactions (TEAR) (xerostomia, dry eyes/skin, constipation, flushing, blurred vision, dizziness, sleep difficulties) have also been reported in children and are often the reason for dose reduction or discontinuation. [7-9] The extended-release (ER) Oxy (OxyER) formulation has been shown to be superior to IR in various studies, [10-12] but its safety and efficacy in children has not been established. [13]

In January 2017, propiverine, used in Europe for many years, was approved by Health Canada for $\mathrm{OAB}$ treatment in adults and children. Other antimuscarinic agents are currently exclusively approved for OAB treatment in adults.

Fesoterodine (Feso) (5-hydroxy-methyltolterodine prodrug, ER antimuscarinic) was approved in Canada in 2012. It has demonstrated significant improvement against placebo in OAB symptoms and quality of life in adults in phase III trials. [14-15, 29] Feso is administered daily (4-8mg tablets).

Given the trivial number of officially approved pharmacological options for children and considering that their use is limited by sub-optimal clinical response or TEAR, there is a need for additional drugs to gain approval by recognized authorities, especially ER formulations to improve compliance and decrease TEAR. Our hypothesis is that Feso and OxyER are safe and effective for the treatment of $\mathrm{OAB}$ in children. Our main objective was to assess the safety of fesoterodine (Toviaz ${ }^{\circledR}$ ) and OxyER (Ditropan XL®) in children with OAB. Our secondary objectives were to compare the short-term efficacy of both medications, and to evaluate the long-term efficacy of Feso. 


\section{Methods}

\section{Study population}

All patients underwent urotherapy before initiating any medication: resolution of constipation, good hydration habits, voiding schedule and optimal voiding position. Anomaly on flow rate electromyography (EMG) (dyssynergia, staccato, etc.) would lead to physiotherapy/biofeedback with bladder retraining for 3-6 months before antimuscarinics are initiated. Prior to inclusion, questionnaire, physical exam, urinalysis/cultures and uroflows/EMG were obtained and normal flow rate index was confirmed.

Patients attending pediatric-urology clinic for LUTS were offered to enter the trial if they fulfilled inclusion and exclusion criteria (Appendix 1). If symptoms did not improve with conservative measures with or without trial of IR Oxy, and less than $65 \%$ of the expected bladder capacity (\%EBC) for age was confirmed $(30+($ age in years $\mathrm{x} 30) \mathrm{mL})$ [1] on a 3-day voiding diary, a single long-acting antimuscarinic (Feso or OxyER) was initiated. Subjects were randomly assigned (1:1) by pharmacy. Patients completing the crossover study were eligible to enrol in a 12-months Feso open-label extension study. Patients having completed the crossover study were eligible to enrol in a 12-month Feso open-label extension study if they demonstrated good tolerance to Feso.

\section{Study design (Figure 1)}

We conducted a single center, prospective, non-inferiority, double-blind, crossover, randomized trial (2015-18). Our main objective was to assess safety of Feso (Toviaz®) and OxyER (Ditropan XL®) in children with OAB. Our secondary objectives were to compare the short-term efficacy of both medications, and evaluate long-term efficacy of Feso in an open-label 12-months extension. Both trials were granted non-objection letters from Health Canada and approved by our Institutional Review Board. Parents provided written consent.

Study power was based on efficacy endpoint. To estimate sample size, we assumed that patients to be included were refractory to conservative treatment (success $<50 \%$ improvement). Sample size was estimated for $2 \times 2$ crossover design for testing non-inferiority, using PASS 13 (Power Analysis and Sample Size Software (2014)). The total sample size was set at $\geq 56$ patients with complete data to achieve $80 \%$ power, one-sided alpha $2.5 \%, 15 \%$ margin of non-inferiority and 0.31 for coefficient of variance. Allowing 10\% dropout (6 patients), we planned to recruit 62 patients.

Prior to and at first visit (V1), children and their parents were again educated on conservative treatment of $\mathrm{OAB}$ (timed voiding, adequate voiding technique, fluid management, and bowel management if necessary) and were instructed to reinforce those measures and to continue them throughout the course of the trial. Study design is detailed in Figure 1 and Appendix 2.

\section{Safety assessment}

The primary endpoint was to assess safety of Feso and OxyER. Safety was evaluated on the basis of TEAR at the end of each crossover phase, and every 4 months during the extension phase, using 
medical history, physical exam, PVR (bladder scan), vital signs, triplicate 12-lead ECG and laboratory parameters.

\section{Efficacy assessment}

The secondary endpoints were to compare the short-term efficacy of Feso and OxyER and to assess the long-term efficacy of Feso. Efficacy was objectively quantified using 3-day voiding diary (VD) at each visit. The primary efficacy variable was change in mean voided volume. Secondary efficacy variables were: urinary frequency, urgency (grade 2-3) and urge incontinence episodes/24hrs. We calculated the relative expected bladder capacity $(\% \mathrm{EBC})$ by dividing mean voided volume on VD by EBC for age. Efficacy was also subjectively evaluated using the patient perception of bladder condition (PPBC) scale (Appendix 3) [16]. Finally, pharmacists calculated medication adherence based on returned pill containers.

\section{Statistical analysis}

Quantitative variables are described as mean $\pm \mathrm{SD}$, and qualitative variables as frequencies and percentages. Parametric (F test or t test) or nonparametric (Kruskal-Wallis, Wilcoxon rank-sum) tests were used to compare continuous data by independent groups after normality verification; chi-square or exact tests used for categorical data comparisons. The McNemar's test was used to compare paired binary data and the Wilcoxon signed-rank test for paired continuous data. To test the primary and secondary crossover outcomes, mixed linear regression models were fitted for continuous outcomes and generalized estimating equation (GEE) logistic models for binary outcomes. Statistical analyses were performed using SAS Statistical Software v.9.4 (SAS Institute, Cary, NC, USA) with a two-sided significance level set at $\mathrm{p}<0.05$.

\section{Results}

The study screened 70 patients and enrolled 62 patients ( 38 boys, 24 girls). Mean age was 8.5 years (range: 5-12). Demographic details are provided in Table 1. We had two early drop-outs (2 boys) not included in the analysis. There were no missed visits and no loss to follow-up.

In the extension phase, we had 26 children. Two children were excluded because they took the medication for only 1 month and then switched back to OxyER for efficacy reasons and 1 child dropped-out after V6 due to anxiety toward blood sampling.

\section{Safety}

There were no life-threatening or serious adverse events during the study. Expected class TEAR were observed such as dry mouth and constipation (Table 2). No statistical difference between the two drugs was demonstrated.

Cardiovascular TEAR were observed but none were severe. There was no change in blood pressure but one patient experienced an increased QTcB interval during the crossover phase. This patient was receiving OxyER and QTcB normalised rapidly on repeated ECG 4 and 12 days later, without treatment. Ten asymptomatic $>20 \%$ elevations of heart rate (HR) were observed in the crossover phase; 9/10 were taking Feso. At baseline, mean HR was 85.4 heartbeat/minute. Mean HR 
after 2 months of treatment with OxyER and Feso was 83.5 and 95.6 beats/min, respectively. From baseline, there was an increase in HR of 10.1 beats/minute $(p<0.01)$ with Feso vs. OxyER $(-1.9$ beats/min, $\mathrm{p}=\mathrm{ns}$ ). Five of these events occurred with Feso 8mg and three with concomitant ADHD medication, and recovered once the dosage was decreased or medication changed. One child had an elevated HR on both antimuscarinics. This patient also had an increase in his ADHD medication during the trial, and this type of side effect is also possible with this class of medication and could explain his HR increase with both medications.

In the extension phase, one patient had QTcB increase and 7 had HR increases, all without symptom. Of these 7 children, four returned to normal HR without intervention, supporting the inherent variability for this parameter in children. Two decreased the dosage and one stopped the medication, HR normalizing afterward.

A total of 15 UTI occurred in 10 different patients during the crossover segment. All of these infections were in female participants ( 7 had prior UTI). There was no difference between the two drugs. One of those patients was excluded from the study because of recurrent UTI with an increased post-void residual. She developed de novo detrusor sphincter dyssynergia not present on initial flow rate. In the 12-months extension phase, there were 5 UTI episodes reported in 3 female participants; two participants also had a UTI in the crossover segment. None had elevated post-void residual.

\section{Efficacy}

The EBC was adjusted to the age of the child at the visit, therefore did compensate for the growth of the child. In general, the normal growth of a child over the 12-months of the study (V1-V7) would mean an increase EBC of $30 \mathrm{~mL}$ between the start $(125 \mathrm{ml})$ to the end $(155 \mathrm{ml})$ of trial, without change in $\% \mathrm{EBC}$. \% EBC mildly improved during the course of the crossover study (Table 3). Using specific patients on the extension segment, we noted an improved \%EBC of almost $10 \%$ over the course of the 12-months.

Changes in $24 \mathrm{~h}$ frequency, urgency and $24 \mathrm{~h}$ urinary incontinence were equivalent between the 2 drugs. The PPBC score was not significantly different between Feso and OxyER (3.5 versus 3.3, respectively; $p=0.12$ ). At the end of the crossover phase, we observed a significant improvement from baseline for each parameter $(\mathrm{p}<0.0001)$ (Table 4$)$.

In the extension phase, parameters were also improved at V7 compared to baseline. The PPBC score was at 3.3 at V7 but seemed to continue to improve at V8, 1 or 2 months after participants had stopped the study medication (2.6).

The adherence to medication remained above $90 \%$.

\section{Post-study data}

To document long-term evolution, we retrieved information on the 34 patients not enrolled for the extension phase. Twenty-three of these 34 children (68\%) are now continent (mean follow-up 18 months). Of the 26 children that enrolled in the extension phase, 23 children completed the 12-month extension phase and 18 are now continent (78\%). Due to lack of improvement in symptoms or \%EBC, 
some patients underwent treatment escalation to dual treatment (antimuscarinic + mirabegron) [21] or switched to mirabegron [22]. Active treatment at last follow-up visit is shown in Table 5.

\section{Discussion}

We present the first prospective, randomized study comparing OxyER to fesoterodine for the treatment of OAB in children. This condition is very bothersome and effective and well-tolerated treatments are needed. Treatment objectives are to improve quality of life by diminishing episodes of urinary incontinence, urgency and by improving bladder capacity. Our double-blind crossover study showed that both, Feso and OxyER, were well-tolerated and safe. Both groups had similar demographics, and efficacy results at the end. Feso and OxyER had similar TEAR profiles and both dosages appeared safe to use in children.

Increase in HR is a well-known class effect of antimuscarinics. The dose-effect correlation on HR elevation was demonstrated in a randomised trial (Feso 4-mg/day and supra-therapeutic 28-mg/day versus placebo). There was a mean HR elevation of 3 beats/minutes for the 4-mg/day dosage and 11 beats/minutes with 28-mg/day dosage. There was no change in QTc measures with either dosage. [26] In a phase 3 placebo-controlled study, the mean HR increase with Feso 4-mg/day and 8-mg/day was 34 beats/minutes and 3-5 beats/minutes. [14] Nitti and al. compared the mean HR change with placebo, 4-mg/day and 8-mg/day. There was a change of 1,3 and 4 beats/minutes respectively. [15] Variation in HR was studied with oxybutynin IR versus placebo and no difference was noted. [17] Similarly to those studies, we observed more HR increase with Feso than with OxyER. It also occurred more often with 8-mg than 4-mg. We noted that some children with elevated HR at 4-mg/day also had concomitant changes in their ADHD medications. Elevation in blood pressure and HR is also well-described for ADHD medications, mostly with amphetamines and atomoxetine. [18] It is possible that these elevations were secondary to ADHD medication or could have been worsened by the simultaneous use of both medications. In conclusion, no significant clinical event occurred because of these HR elevations. In fact, a systematic review on the subject by Fleming et al. in Lancet confirmed that at a mean age of 8 years (patients in our study), the mean HR is 90 beats $/ \mathrm{min}$ and the $99^{\text {th }}$ percentile is at 120 beats/min. [30] Therefore, our patients' HR did fall within normal limits but exceeded the limit we set in the protocol ( $\leq 20 \%$ increase from baseline) for safety reasons. Similar to the literature, we had no concern about QTcB changes with the 2 drugs. Nonetheless, we would suggest a closer cardiac monitoring of children being on both ADHD meds and fesoterodine.

Patients presented UTI during the study and they occurred only in girls. There was no difference in UTI rates between the two drugs. Similarly to the current literature, the incidence of UTI does not seem to be increased from the general children population by either Feso or OxyER. [15][19]

As OAB is sometimes associated with ADHD [23-24], Feso seemed to be an interesting choice of medication since it has very low likelihood of crossing the blood-brain barrier. [27] We had a low incidence of central nervous system adverse event in our study. OxyER had a tendency toward more events of irritability and insomnia over Feso $(\mathrm{p}=\mathrm{ns})$ (table 2). To support this observation, in a phase I, 4-treatment, crossover, double-blind, placebo- and positive-controlled study in elderly healthy 
volunteers, there was no difference in cognitive impairment between placebo and Feso. [20] Another study evaluating cognitive impact of antimuscarinics, Oxy was associated with more cognitive impairment than other antimuscarinics. [25] Evidently, our trial was not designed to study this outcome specifically but further research on the subject would be interesting.

$\%$ EBC improved over time. The major factor in improving bladder capacity with antimuscarinics has been associated with treatment duration. Using \%EBC automatically corrects $\mathrm{EBC}$ according to the age of the patients at a given time. Normal growth would keep the EBC stable over time. In a study of 27 children with daytime incontinence that switched from oxybutynin IR to OxyER, there was an improvement of $15 \%(\mathrm{p}<0.01)$ in $\% \mathrm{EBC}$. [10] In our study, on top of the improvement in urge and urge incontinence, we had 10\% improvement of \%EBC after one year of treatment on fesoterodine (V2-V7), reflecting the potential efficacy of the medication.

There was no significant statistical or clinical difference in efficacy between the two drugs. There was an improvement from baseline with all variables with both medications. In the extension phase, the improvement was progressive. It was interesting to see that even though the mean voided volume deteriorated between V7 and V8 (11 of the 24 children either stopped or decreased the dosage of their medication before V8), the PPBC score improved. We think that children who stopped their medication probably had a better perception of their condition being off medication and having fewer side effects, making their quality of life better.

Beyond the study period, most children became completely continent (69\%) and a few were able to taper off medication (33\%) (Table 5). The most important thing that we get from these data is that $\mathrm{OAB}$ is a chronic condition that necessitates an extended medical follow-up, sometimes attempting several approaches/drugs before finding the one that fulfills the patient's needs. Conservative measures need to be reinforced at every visit, and compliance to those measures and medication, need to be verified. [28] Any modification in concomitant medication must be taken into account regarding pharmacologic interactions and potential new side effects, especially for patients with ADHD.

\section{Limitations}

Although we performed a prospective, randomized double-blind study, the trial remains of moderate size for pediatric $\mathrm{OAB}$, as it was designed as a non-inferiority study. Also, the trial was a single-center study; a multicenter trial would have helped making the findings more generalizable. We did not include a placebo-controlled group nor made a comparison with oxy IR. In addition, we could have included an extension phase for OxyER but we report on their outcome (table 5). The extension phase was only offered to children responding to fesoterodine, this could have introduced a selection bias contributing to the improvement of PPBC scores. 


\section{Conclusions}

The use of fesoterodine or OxyER appears to be safe and effective treatment options for $\mathrm{OAB}$ in children. The efficacy of both drugs was similar. Based on the 12-months extension phase and beyond, $\mathrm{OAB}$ in children needs long-term treatment to achieve the ultimate goal of urinary continence.

\section{Take-home message}

Long-acting formulations of oxybutynin and fesoterodine are effective in the treatment of overactive bladder in pediatrics as they improve median voided volume, quality of life and number of incontinence episodes with few side effects. 


\section{References}

1. Austin PF, Bauer SB, Bower W, et al. The standardization of terminology of lower urinary tract function in children and adolescents: Update report from the standardization committee of the International Children's Continence Society. Neurourol Urodyn 2016 Apr;35(4):471-81.

2. Feldman AS, Bauer SB. Diagnosis and management of dysfunctional voiding. Curr Opin Pediatr 2006 Apr;18(2):139-47.

3. Kajiwara M, Inoue K, Kato M, et al. Nocturnal enuresis and overactive bladder in children: an epidemiological study. Int J Urol 2006 Jan;13(1):36-41.

4. Chung JM, Lee SD, Kang DI, et al. Prevalence and associated factors of overactive bladder in Korean children 5-13 years old: a nationwide multicenter study. Urology 2009 Jan;73(1):63-7.

5. Hägglöf B, Andrén O, Bergström E, et al. Self-esteem in children with nocturnal enuresis and urinary incontinence improvement of self-esteem after treatment. Eur Urol 1998;33 Suppl 3:169.

6. Gormley EA, Lightner DJ, Burgio KL, et al. Diagnosis and treatment of overactive bladder (non-neurogenic) in adults: AUA/SUFU guideline. J Urol 2012 Dec;188(6 Suppl):2455-63.

7. Massad CA, Kogan BA, Trigo-Rocha FE. The pharmacokinetics of intravesical and oral oxybutynin chloride. J Urol 1992 Aug;148(2 Pt 2):595-7.

8. Kasabian NG, Vlachiotis JD, Lais A, et al. The use of intravesical oxybutynin chloride in patients with detrusor hypertonicity and detrusor hyperreflexia. J Urol 1994 Apr;151(4):944-5.

9. Alloussi S, Mürtz G, Braun R, et al. Efficacy, tolerability and safety of propiverine hydrochloride in comparison to oxybutynin in children with urge incontinence due to overactive bladder: Results of a multicentre observational cohort study. BJU Int 2010 Aug;106(4):550-6.

10. Van Arendonk KJ, Knudson MJ, Austin JC, et al. Improved efficacy of extended release oxybutynin in children with persistent daytime urinary incontinence converted from regular oxybutynin. Urology 2006 Oct;68(4):862-5.

11. Youdim K, Kogan BA. Preliminary study of the safety and efficacy of extended-release oxybutynin in children. Urology 2002 Mar;59(3):428-32.

12. Reinberg Y, Crocker J, Wolpert J, et al. Therapeutic efficacy of extended release oxybutynin chloride, and immediate release and long acting tolterodine tartrate in children with diurnal urinary incontinence. J Urol 2003 Jan;169(1):317-9.

13. Ditropan XL® Product Monograph. Products. Janssen Inc. Toronto, Ont. Available at: https://www.janssen.com/canada/sites/www_janssen_com_canada/files/prod_files/live/ditropan cpm.pdf. Accessed September 6, 2018.

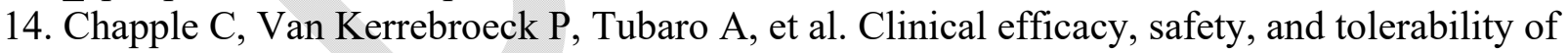
once-daily fesoterodine in subjects with overactive bladder. Eur Urol 2007 Oct;52(4):1204-12.

15. Nitti VW, Dmochowski R, Sand PK, et al. Efficacy, safety and tolerability of fesoterodine for overactive bladder syndrome. J Urol 2007 Dec;178(6):2488-94.

16. Coyne KS, Matza LS, Kopp Z, et al. The validation of the patient perception of bladder condition (PPBC): a single-item global measure for patients with overactive bladder. Eur Urol 2006 Jun;49(6):1079-86.

17. Chapple CR, Abrams P. Comparison of darifenacin and oxybutynin in patients with overactive bladder: assessment of ambulatory urodynamics and impact on salivary flow. Eur Urol 2005; 48: $102-9$.

18. Hennissen L, Bakker MJ, Banaschewski T, et al; ADDUCE consortium. Cardiovascular Effects of Stimulant and Non-Stimulant Medication for Children and Adolescents with ADHD: A 
Systematic Review and Meta-Analysis of Trials of Methylphenidate, Amphetamines and Atomoxetine. CNS Drugs. 2017 Mar;31(3):199-215

19. Kraus SR, Ruiz-Cerda JL, Martire D, et al. Efficacy and tolerability of fesoterodine in older and younger subjects with overactive bladder. Urology 2010;76(6):1350-7.

20. Kay $\mathrm{GG}^{1}$, Maruff P, Scholfield D, et al. Evaluation of cognitive function in healthy older subjects treated with fesoterodine. Postgrad Med. 2012 May;124(3):7-15.

21. Morin F, Blais AS, Nadeau G, et al. Dual Therapy for Refractory Overactive Bladder in Children: A Prospective Open-Label Study. J Urol. 2017 Apr;197(4):1158-1163

22. Blais AS, Nadeau G, Moore K, et al. Prospective Pilot Study of Mirabegron in Pediatric Patients with Overactive Bladder. Eur Urol. 2016 Jul;70(1):9-13

23. Burgu B, Aydogdu O, Gurkan K, et al. Lower urinary tract conditions in children with attention deficit hyperactivity disorder: correlation of symptoms based on validated scoring systems. $\mathrm{J}$ Urol. 2011 Feb;185(2):663-8

24. Yousefichaijan P, Sharafkhah M, Rafiei M, et al. Attention-deficit/hyperactivity disorder in children with overactive bladder; a case-control study. J Renal Inj Prev. 2016 Aug 9;5(4):193-9

25. Kay, G. G., Ebinger, U.: Preserving cognitive function for patients with overactive bladder: evidence for a differential effect with darifenacin. Int J Clin Pract, 62: 1792, 2008

26. Malhotra B, Wood N, Sachse R, et al. Thorough QT study of the effect of fesoterodine on cardiac repolarization. Int J Clin Pharmacol Ther. 2010 May;48(5):309-18

27. Ernesto C, Malhotra B, Bungay PJ, et al. A comprehensive nonclinical evaluation of the CNS penetration potential of antimuscarinic agents for the treatment of overactive bladder. $\mathrm{Br} \mathrm{J}$ of Clin Pharm

28. Fortin A, Morin V, Ramsay S, et al. Adherence to antimuscarinics in children with overactive bladder. Paediatr Child Health. 2017 Aug;22(5):255-258. doi: 10.1093/pch/pxx055. Epub 2017 May 17.

29. Wagg A, Arumi D, Herschorn S, et al . A pooled analysis of the efficacy of fesoterodine for the treatment of overactive bladder, and the relationship between safety, co-morbidity and polypharmacy in patients aged 65 years or older. Age Ageing. 2017 Jul 1;46(4):620-626. doi: 10.1093/ageing/afw252.

30. Fleming S, Thompson M, Stevens R, et al. Normal ranges of heart rate and respiratory rate in children from birth to 18 years of age: a systematic review of observational studies. Lancet. 2011 Mar 19;377(9770):1011-8. doi: 10.1016/S0140-6736(10)62226-X 


\section{Figures and Tables}

Fig. 1. Study design.
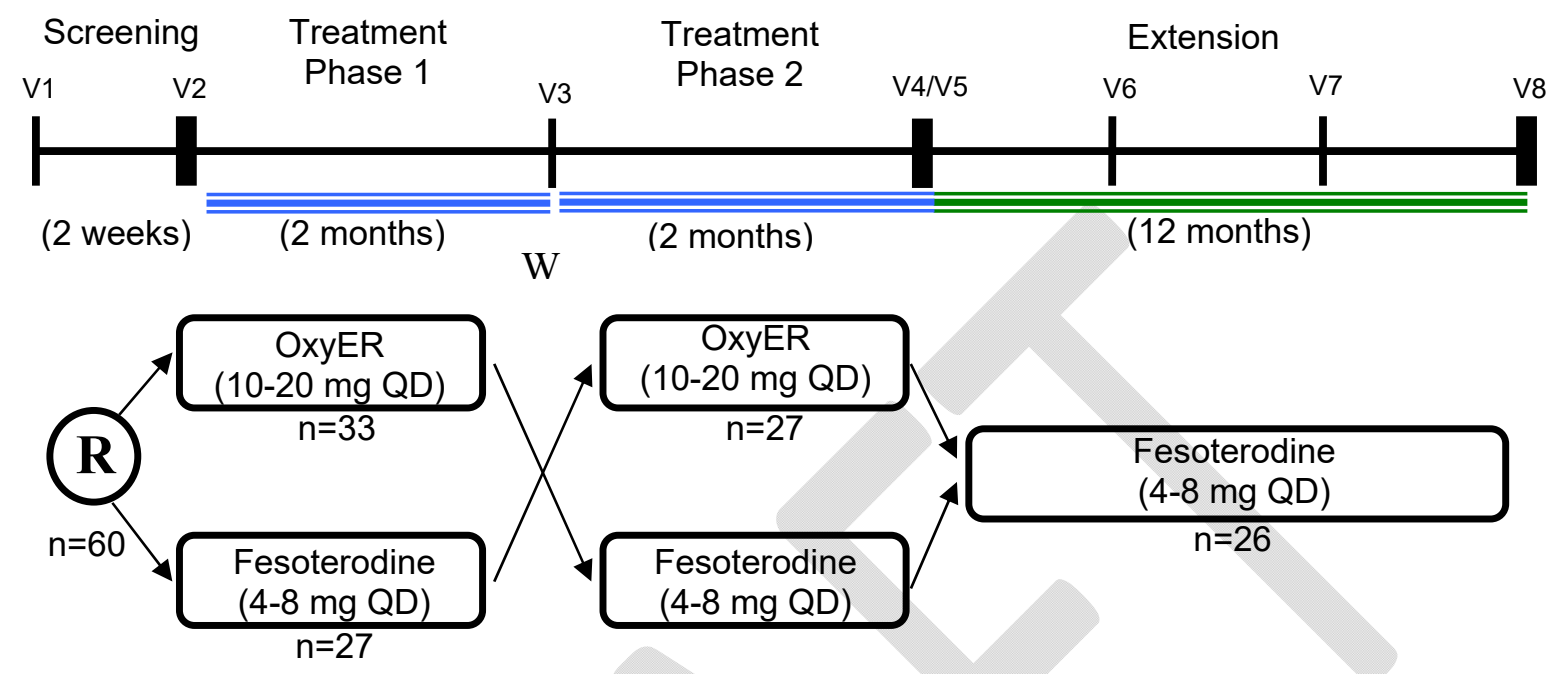

R: randomization; V: visit

$\equiv$ : Crossover period

$\overline{\overline{\bar{V}}}$ : Extension period on fesoterodine

V1-V2: Washout from any antimuscarinics.

V2: patients were randomized in a 1:1 ratio to Group Feso-Oxy or Group Oxy-Feso.

V3: 3-day washout period prior to second medication. (W)

V4: patients/parents were asked to identify their favourite treatment phase.

If they chose fesoterodine, they could enter the extension phase if they wanted.

See Appendix 2 for details 
Table 1. Study participants' description randomized in the crossover phase and included in the 12-months open-label extension on fesoterodine

\begin{tabular}{|l|c|c|c|c|c|}
\hline & \multicolumn{3}{|c|}{$\begin{array}{c}\text { Crossover study } \\
\text { Mean } \pm \text { SD } \\
\text { n=60* }\end{array}$} & $\begin{array}{c}\text { Extension phase } \\
\text { Mean } \pm \text { SD } \\
\text { n=26 }\end{array}$ \\
\cline { 2 - 5 } & $\begin{array}{c}\text { Groupe 1 } \\
\text { (oxy-feso) } \\
\mathbf{n = 3 3}\end{array}$ & $\begin{array}{c}\text { Groupe 2 } \\
\text { (feso-oxy) } \\
\text { n= 27 }\end{array}$ & $\mathbf{p}$ & Total & \\
\hline Male (\%) & $19(57.6 \%)$ & $17(63 \%)$ & 0.79 & $36(60 \%)$ & $17(65 \%)$ \\
\hline Age, (year) & $8.5 \pm 1.8$ & $8.4 \pm 1.9$ & 0.95 & $8.5 \pm 1.8$ & $8.8 \pm 2.2$ \\
\hline ADHD medication & $6(18.2 \%)$ & $2(7.4 \%)$ & 0.28 & 8 & 5 \\
\hline Weight, (kg) & $29.3 \pm 8.9$ & $28.1 \pm 7.3$ & 0.8 & $28.8 \pm 8.2$ & $29.0 \pm 9.8$ \\
\hline Height, $(\mathrm{m})$ & $1.31 \pm 0.14$ & $1.29 \pm 0.1$ & 0.74 & $1.30 \pm 0.12$ & $1.32 \pm 0.14$ \\
\hline BMI, $\mathrm{kg} / \mathrm{m}^{2}$ & $16.6 \pm 2$ & $16.6 \pm 2.4$ & 1.00 & $16.6 \pm 2.2$ & $16.6 \pm 2.0$ \\
\hline
\end{tabular}

*62 randomized patients. 70 patients were screened, two children were excluded from analysis: 1 did not initiate the treatment and the second did not complete the first phase. 58 children completed the crossover phase of the trial (self-exclusion [1], recurrent urinary tract infection [1] between V3 and V4). ADHD: attention deficit and hyperactivity disorder; BMI: body mass index; SD: standard deviation. 


\begin{tabular}{|c|c|c|c|c|c|}
\hline \multirow[b]{2}{*}{ Body system } & \multicolumn{3}{|c|}{ Crossover phase } & \multicolumn{2}{|c|}{$\begin{array}{c}\text { Extension } \\
\text { phase }\end{array}$} \\
\hline & $\begin{array}{c}\text { Total } \\
(\%)\end{array}$ & $\begin{array}{c}\text { Fesoterodine } \\
\text { n=events }\end{array}$ & $\begin{array}{c}\text { OxyER } \\
\text { n= events }\end{array}$ & $\begin{array}{c}n= \\
\text { events }\end{array}$ & $\begin{array}{c}\text { Total } \\
(\%)\end{array}$ \\
\hline \multicolumn{6}{|l|}{ Cardiovascular } \\
\hline Variation blood pressure & 0 & 0 & 0 & 1 & 3.8 \\
\hline Variation in heart rate $(\geq 20 \%)$ & 14.5 & $9^{3}$ & 1 & $7^{4}$ & 26.9 \\
\hline Increased QTcB & 1.6 & 0 & 1 & 1 & 3.8 \\
\hline Flushing (skin, cheeks) & 3.2 & 2 & 0 & 0 & 0 \\
\hline \multicolumn{6}{|l|}{ Genitourinary } \\
\hline Urinary tract infection & 16.1 & 7 & 8 & 5 & 11.5 \\
\hline Increased PVR & 1.6 & 0 & 1 & 0 & 0 \\
\hline \multicolumn{6}{|l|}{ Gastrointestinal } \\
\hline Nasal bleeding/epistaxis & 6.4 & 2 & 2 & 3 & 1.1 \\
\hline Dry mouth/eyes/hands & 43.5 & 17 & 17 & 11 & 42.3 \\
\hline Constipation & 13.3 & 5 & 3 & 5 & 19.2 \\
\hline Diarrhea & 8.0 & 1 & 4 & 0 & 0 \\
\hline Abdominal pain & 20.9 & 8 & 7 & 6 & 23.0 \\
\hline Appetite modification & 8.0 & 2 & 4 & 0 & 0 \\
\hline Nausea/vomiting & 4.8 & + & 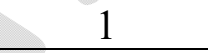 & 2 & 7.6 \\
\hline Variation AST/ALT value $^{1}$ & 1.6 & 1 & 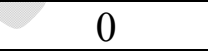 & 1 & 3.8 \\
\hline \multicolumn{6}{|l|}{ Nervous system $^{2}$} \\
\hline Headache & 3.2 & 2 & 0 & 3 & 1.1 \\
\hline Irritability & 6.4 & 2 & 3 & 4 & 15.3 \\
\hline Insomnia & 1.6 & 0 & 1 & 0 & 0 \\
\hline
\end{tabular}

${ }^{1}$ Mild elevation at V4. One month later, the control was normal without intervention. ${ }^{2} \mathrm{O}$ t the 6 children that experienced either irritability or insomnia, two had ADHD. ${ }^{3}$ Five of the HR increase occurred with Feso $8 \mathrm{mg}$ and three with concomitant ADHD medication, and recovered once Feso dosage was decreased or changed to OxyER at crossover. ${ }^{4} \mathrm{Six}$ of the 7 children with HR increase in extension phase were on Feso $8 \mathrm{mg}$. One on $4 \mathrm{mg}$ also had increase in the dosing of his ADHD medication. AST/ALT: aspartate transaminase/alanine transaminase; PVR: post-void residual. 


\begin{tabular}{|l|c|c|c|c|c|c|}
\hline Table 3. Functional bladder capacity \\
\hline Period & \multicolumn{2}{|c|}{ Baseline } & \multicolumn{2}{|c|}{$\begin{array}{c}\text { End of crossover V4 } \\
(\mathbf{n}=\mathbf{6 0})\end{array}$} & \multicolumn{2}{c|}{$\begin{array}{c}\text { Extension phase } \mathbf{V 7}^{\mathbf{2}} \\
(\mathbf{n}=\mathbf{2 6})\end{array}$} \\
\hline \multirow{3}{*}{$\begin{array}{l}\text { Bladder } \\
\text { capacity }\end{array}$} & $\begin{array}{c}\text { Mean volume } \\
(\mathrm{mL})\end{array}$ & $\% \mathrm{EBC}^{1}$ & $\begin{array}{c}\text { Mean volume } \\
(\mathrm{mL})\end{array}$ & $\begin{array}{c}\text { Mean volume } \\
(\mathrm{mL})\end{array}$ & $\% \mathrm{EBC}$ \\
\cline { 2 - 7 } & 125 & 44.5 & 146 & 50.0 & 171 & $53.9^{3}$ \\
\hline
\end{tabular}

${ }^{1} \mathrm{EBC}$ was adjusted to the age of the child at each visit to compensate for the growth of the child ([age (years) + 1] x $30 \mathrm{~mL}$ ). [1] The normal growth of a child over the 12-months of the study (V1-V7) would mean an increase EBC of $30 \mathrm{~mL}$ between baseline $(125 \mathrm{ml})$ to the end of trial, without change in $\% \mathrm{EBC}$. ${ }^{2} \% \mathrm{EBC}$ was calculated at $\mathrm{V} 7$ because in the last four months of the extension phase, participants had the opportunity to decrease and stop the medication and consequently some patients could present worsening of their bladder capacity at V8, underestimating the gain in capacity.3: Comparing baseline $\% \mathrm{EBC}$ vs. end of extension phase $(\mathrm{n}-26), \mathrm{p}<0.05 . \% \mathrm{EBC}$ : relative expected bladder capacity for age

\begin{tabular}{|c|c|c|c|c|c|c|}
\hline \multirow{2}{*}{\multicolumn{5}{|c|}{ Table 4. Efficacy variables }} & \multirow{2}{*}{\multicolumn{2}{|c|}{ 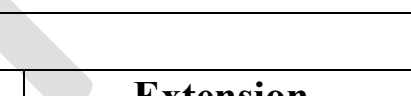 }} \\
\hline & & & & & & \\
\hline & Baseline & Fesoterodine & OxyER & $\begin{array}{c}\text { End of } \\
\text { crossover } \\
(\mathrm{V} 4) \mathbf{n}=\mathbf{6 0}\end{array}$ & $\begin{array}{c}\mathrm{V7} \\
\mathrm{n}=26 \\
\end{array}$ & $\begin{array}{c}\mathrm{V8} \\
\mathrm{n}=26 \\
\end{array}$ \\
\hline $\begin{array}{l}\text { Mean voided volume per } \\
\text { micturition, }(\mathrm{mL})(\mathrm{SD})\end{array}$ & $125(53)$ & $147(56)^{*}$ & $156(56)^{*}$ & $146(53)^{*}$ & $171(74)^{*}$ & $160(90)^{\#}$ \\
\hline $\begin{array}{l}\text { Urinary frequency per } \\
24 \mathrm{~h}, \text { mean }(\mathrm{SD})^{\sharp}\end{array}$ & $6.8(2.4)$ & $6.0(2.2)^{*}$ & $5.9(2.2)^{*}$ & $5.9(2.6)^{*}$ & $5.8(1.5)^{*}$ & $6.2(2.2)^{\#}$ \\
\hline $\begin{array}{l}\text { Urinary incontinence per } \\
24 \mathrm{~h}, \text { mean }(\mathrm{SD})\end{array}$ & $1.8(1.7)$ & $1.0(1.1)^{*}$ & $0.9(1.1)^{*}$ & $0.9(1.2)^{* 1}$ & $1.2(1.7)^{*}$ & $1.2(2.1)^{*}$ \\
\hline $\begin{array}{l}\text { Grade } 2-3 \text { urgency } \\
\text { episodes per } 24 \mathrm{~h} \text {, mean } \\
\text { (SD) }\end{array}$ & $1.7(2.3)$ & $1.0(1.4)^{*}$ & $1.1(1.4)^{*}$ & $1.0(1.5)^{*}$ & $1.4(2.3)^{\#}$ & $1.3(2.1)^{\#}$ \\
\hline PPBC score (SD) & $3.6(1.1)$ & $3.5(1.2)^{\#}$ & $3.3(1.2)^{*}$ & $3.4(1.2)^{*}$ & $3.2(1.4)^{*}$ & $2.6(1.3)^{*}$ \\
\hline
\end{tabular}

The primary efficacy variable was the change in mean voided volume, calculated using the 3 -day VD, excluding the morning void. "Secondary efficacy variables, calculated with the 3-day VD.

Efficacy was also subjectively measured using the patient perception of bladder condition (PPBC) scale (Appendix 2) [17] at baseline, at the end of each crossover phase, and every 4 months during the extension phase. ${ }^{*}$ Comparison from baseline to end of crossover $(n=60)$ or end of extension phase $(\mathrm{n}=26), \mathrm{p}<0.0001 .{ }^{\#}$ Comparison from baseline to end of crossover $(\mathrm{n}=60)$ or end of extension phase $(n=26), p<0.01 .{ }^{1}$ At the end of the crossover phase, 28 patients achieved daytime continence (15 Feso, 13 OxyER). SD: Standard deviation 


\begin{tabular}{|c|c|c|c|}
\hline & & $\begin{array}{c}\text { Participants in the } \\
\text { crossover study only }{ }^{1} \\
n=34\end{array}$ & $\begin{array}{c}\text { Participants in the } \\
\text { extension phase }{ }^{2} \\
n=26\end{array}$ \\
\hline \multicolumn{2}{|l|}{ Gender (M/F) } & $19 / 15$ & $17 / 9$ \\
\hline \multicolumn{4}{|c|}{ Active treatment at last visit } \\
\hline & None & 12 & 9 \\
\hline & OxyER & 11 & 2 \\
\hline & Fesoterodine & 0 & 5 \\
\hline & Mirabegron & 4 & 3 \\
\hline & Dual treatment ${ }^{*}$ & 6 & 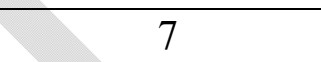 \\
\hline & Lost to followup & 1 & 0 \\
\hline \multicolumn{2}{|l|}{ Continent } & $23(68 \%)$ & $18(69 \%)$ \\
\hline
\end{tabular}

${ }^{1}$ Patients not participating to the extension phase, therefore ending at Visit 4 , active treatment after the study if symptoms persisted. 34 of the 60 patients. Mean follow-up 18 months. ${ }^{2}$ Patients who were on the 12-month extension phase with fesoterodine, active treatment after Visit 8 if symptoms persisted. 26 remaining patients of the initial 60 patients. Mean followup 9 months. *Dual: antimuscarinic plus mirabegron [25]. 


\section{Appendix 1. Inclusion/exclusion criteria}

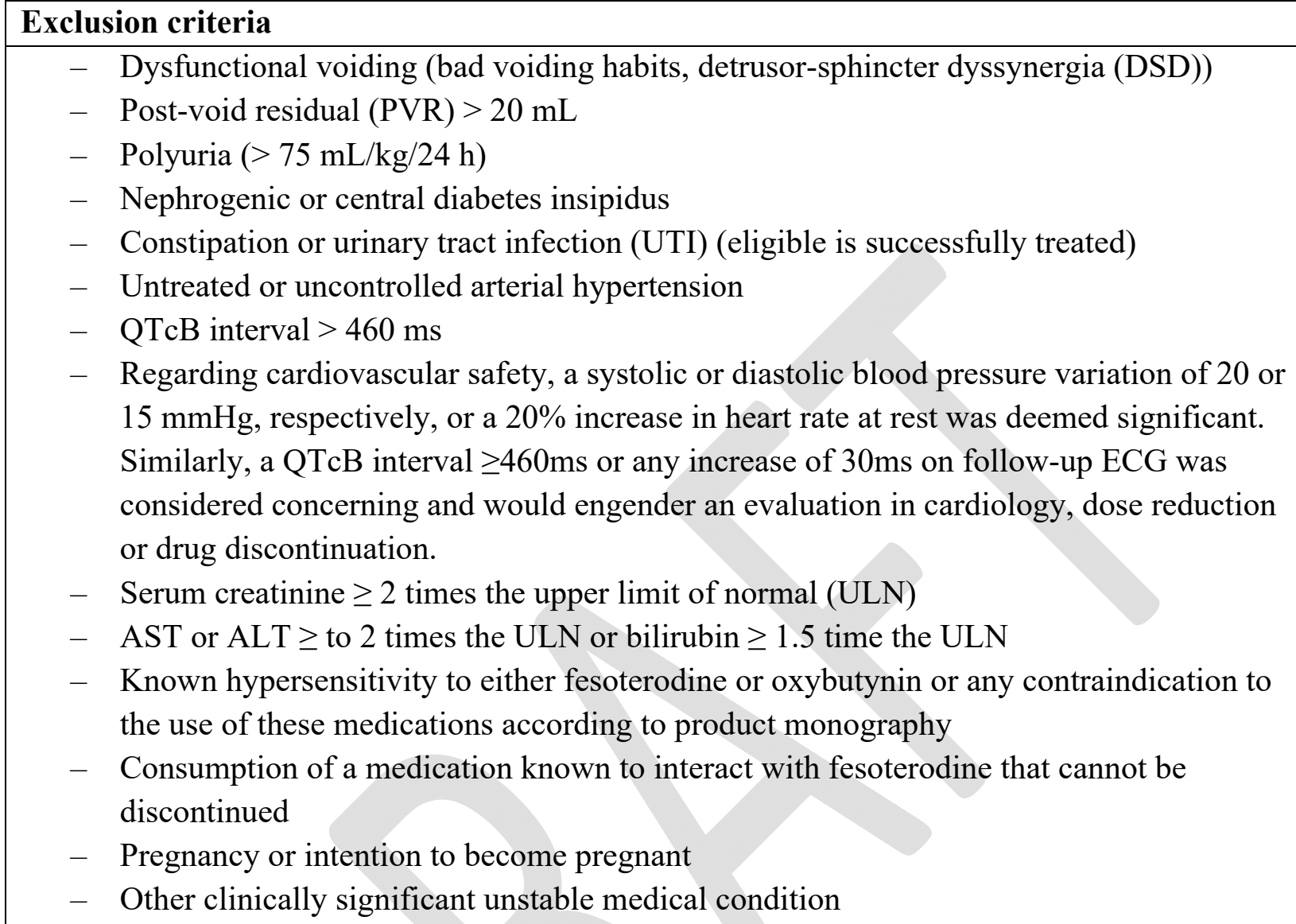

- Dysfunctional voiding (bad voiding habits, detrusor-sphincter dyssynergia (DSD))

- Post-void residual (PVR) $>20 \mathrm{~mL}$

- Polyuria (>75 mL/kg/24 h)

- Nephrogenic or central diabetes insipidus

- Constipation or urinary tract infection (UTI) (eligible is successfully treated)

- Untreated or uncontrolled arterial hypertension

- QTcB interval > $460 \mathrm{~ms}$

- Regarding cardiovascular safety, a systolic or diastolic blood pressure variation of 20 or $15 \mathrm{mmHg}$, respectively, or a $20 \%$ increase in heart rate at rest was deemed significant. Similarly, a QTcB interval $\geq 460 \mathrm{~ms}$ or any increase of $30 \mathrm{~ms}$ on follow-up ECG was considered concerning and would engender an evaluation in cardiology, dose reduction or drug discontinuation.

- Serum creatinine $\geq 2$ times the upper limit of normal (ULN)

- $\mathrm{AST}$ or ALT $\geq$ to 2 times the ULN or bilirubin $\geq 1.5$ time the ULN

- Known hypersensitivity to either fesoterodine or oxybutynin or any contraindication to the use of these medications according to product monography

- Consumption of a medication known to interact with fesoterodine that cannot be discontinued

- Pregnancy or intention to become pregnant

- Other clinically significant unstable medical condition

\section{Inclusion criteria}

- $\quad$ Male or female $\geq 5$ years old and $\leq 14$ years old

- OAB diagnosis according to the International Children Continence Society (ICCS) and less than $65 \%$ of the expected mean bladder capacity for age is confirmed ([age (years) +1 ] x $30 \mathrm{~mL}$ ) on a 3-day voiding diary.

- Weight and height are within the normal percentile (3rd to 97th percentile) and weight is $\geq 20 \mathrm{~kg}$ (3rd percentile of a 8 y.o. child, boy or girl), according to the CDC growth chart

- Ability to swallow pills

- Subjects/parents (vs. legal guardian) agree to participate to the following study and sign the informed consent/assent.

- Normal flow rate index.

- Subjects/parents (or legal guardian) are able to comply with the study requirements and with the medication restrictions.

- Female subjects of childbearing potential must have a negative serum or urine 
pregnancy test at enrollment and must agree to maintain highly effective birth control during the study. Sexually active male subjects agree to use a barrier method of birth control with female partner for the duration of the study and at least one month after ending study treatment. Sexually active male subjects agree to use a condom for the duration of the study and for at least one month after ending study treatment and the female partner to use a reliable form of birth control for the duration of the study and for at least one month after ending study treatment. 


\section{Appendix 2. Details on study methods}

- V1-V2: Washout from any antimuscarinics. Normal flow rate index confirmed. Confirm conservative $\mathrm{OAB}$ treatment (timed voiding, adequate voiding technique, fluid management, and bowel management if necessary).

- Safety measures at every V: vital signs (blood pressure, heart rate, triplicate 12-lead ECG (QTcB), and urinalysis.

- Laboratory parameters (complete blood count, renal and liver function tests) at V1, 3, 4 and 7.

- V2: patients were randomized in a 1:1 ratio to Group Feso-Oxy or Group Oxy-Feso.

- 3-day Voiding diary (VD) provided at each visit.

- V3: 3-day washout period prior to second medication.

- At mid-course of each phase (after 4 weeks), parents were offered the possibility to double the medication dose (Feso 8mg or Oxy 20mg QD) during a telephone interview with the research nurse.

- V4: patients/parents were asked to identify their favourite treatment phase.

- If they chose fesoterodine, they could enter the extension phase if they wanted. For patients that entered the extension study, the last visit of the crossover study (V4) would also serve as the first visit of the extension study (V5- time- 4 months).

- At V5, patients from Group Feso-Oxy restarted Feso at the last well-tolerated dose, and patients from Group Oxy-Feso continued Feso at the same dose.

- V6: dose increase was again discussed if the patient was still on the 4-mg dose.

- V6, V7, and V8 occurred at 4-month intervals after V5.

- If patients experienced significant TEAR or if the patient was dry (no incontinence), without grade 2 or 3 urgency episodes, and with $\% \mathrm{EBC}$ consistently $\geq 75 \%$ on $\mathrm{VD}$, dose tapering or cessation was considered.

- If dose tapering was initiated at V6, a nurse contacted the parents one month after the changes to either return to a higher dose or to consider medication cessation. At V7, dose tapering was initiated for one month, followed by medication cessation, unless the patient was still symptomatic. In that case, dose tapering was to be initiated two months after V7, followed by medication cessation one month later. Therefore, every patient had attempted medication cessation prior to V8.

\section{Appendix 3. The patient perception of bladder condition (PPBC) scale}

\begin{tabular}{|l|l|}
\hline My bladder condition: & \\
\hline 1 & Does not cause me any problems at all \\
\hline 2 & Causes me some very minor problems \\
\hline 3 & Causes me some minor problems \\
\hline 4 & Causes me (some) moderate problems \\
\hline 5 & Causes me severe problems \\
\hline 6 & Causes me many severe problems \\
\hline
\end{tabular}

